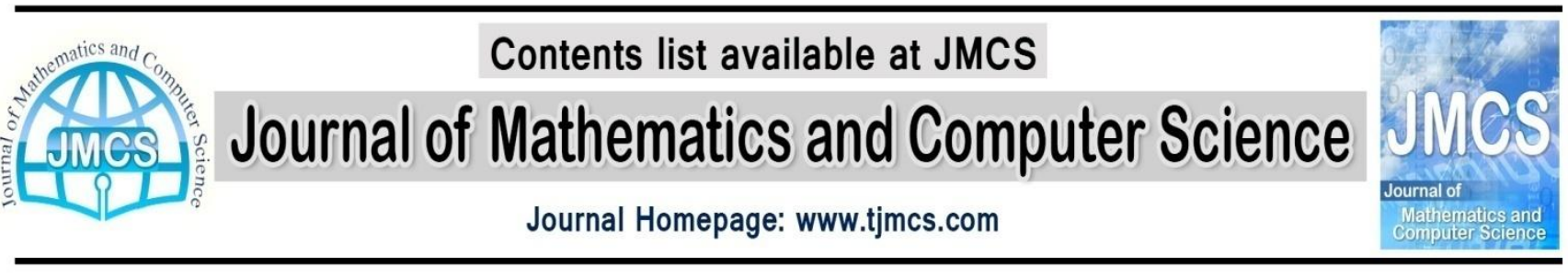

\title{
Noise Detection and Reduction Using Algorithms Based on Particle Swarm Optimization and Fuzzy Decision Making
}

\author{
payiz1380@yahoo.com \\ azadi_1983@yahoo.com
}

Mohammadreza Heydari Nezhad, Azar Azadi

Islamic Azad University of Qeshm, International branch

Article history:

Received December 2013

Accepted January 2014

Available online January 2014

\begin{abstract}
Noise filtering has a special importance for systems related to digital images processing. Mostly conventional filter used in image processing applications for noise cancellation is the median filter which is especially used to reduce salt and pepper noise in images. However median filter has a good performance in smoothing and noise reduction, but leads to blurring and elimination of some fine details and destruction of edges in image. One approach in confronting with conventional noise filters like median filter is not to operate same process on all pixels. This means by using a decision block or classifier, confronting with pixels must be determined appropriately. In this thesis we address to the problem of designing such decision blocks for noise reduction based on fuzzy inference theory. Another main subject in this thesis is the procedure of utilization of optimization algorithms based on swarm movement of particles for designing the fuzzy part.
\end{abstract}

Keywords:Noise Filtering, Fuzzy Set Theory, Particle Swarm Optimization

\section{Introduction}

A major problem of the output images in Cameras is noise which is resulted from a random signal over a signal or a main image, this random signal is capable of influencing on the main signal in two modes of convolution and/or addition. The median Filter is normally used for processing images in order to reduce noise.

Considering a defined neighborhood around each pixel, It attributes the luminance intensity of this neighborhood to the central pixel. This contributes to smoothing the image and reduction of such noises as salt-pepper noise. Given the notion that the noise is more capable at higher frequency 
components, these methods perform as compared to image smoothing. Therefore, while applying these filters, some characteristics of higher Frequency of the main image like image edges will be removed or destructed. In order to remove the problems of these methods of noise reduction, different recommendations have been provided which included adaptive Filters or weighted Filters in place of simple median filter.

This article considers the Fuzzy decision maker systems[1-4] optimized by particle swarm optimization (PSO) algorithm[5-6] for identifying and reducing image noise. In the recommended method, the image pixels are not treated in the same manner. However, the presence and intensity of noise is firstly identified. In this regard, the pixels are divided into different categories and the manner and intensity of Filtering process differs for each of them. The Fuzzy decision maker block accounts for identifying the noise pixels using the Fuzzy logic makes classified and non-binary decision makings possible. Therefore, a noise membership rank could be used to select or reject a pixel as a noisy one. [9]

In order to design a Fuzzy system, no exact or recognized method exists and the defining parameters of a Fuzzy inference system are either determined by trial and error or by using an optimization method, proper values are determined for its parameters. The recommended methods of this research are based on an algorithm called particle swarm optimization. PSO is used here and their efficiency will be compared. The functions of PSO are based on Mass Movement of the particles in a setting of optimization variables and there is a tendency to select places with better Fitting.[11] The movements of particles within the space of the solutions of the problem are as follows:

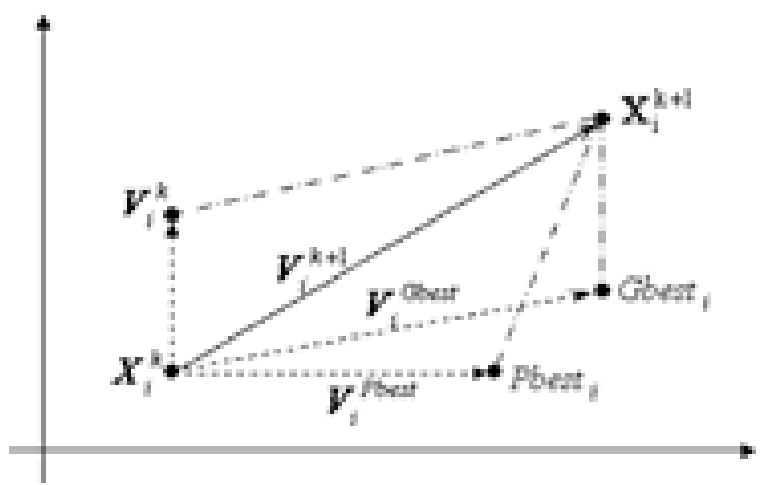

Figure 1: movements of particles within the space of the solutions for the problem.

As it can be seen, the next place of the particle $\left(x_{i}^{k+1}\right)$ is determined by considering its tendency to move toward the best existing solution in the gross memory of the particles $\left(G_{\text {best }}\right)$ and the best solution in particle's private memory $\left(P_{\text {best }}\right)$ using PSO for optimization leads to quicker Function.[7,10,12] 


\section{Modeling fuzzy noise filter}

Here a new kind of salt and pepper Filter called as noise adapted Fuzzy switching median Filter (NAFSM) will be introduced. Indeed, it is a mixture of the simple adaptive median Filter and Fuzzy switching median Filter.

The adaptive behavior of NAFSM made it capable of expanding the size of Filter's window with respect to noise intensity. Thus, a severe salt and pepper noise could be filtered. Therefore, the intrinsic behavior of the Median's switching can speed up Filtering operation which also maintaining the details of the image. In addition, the Fuzzy inference is related to uncertainty of the image's local information and it helps creation of correct sentences by restoring identified noisy pixels.

The NAFSM is a two stage recursive Filter. At first, the intensity of the salt and pepper noise will be determined before the place of the noisy pixel could be recognized. The stage of Filtering begins after the noisy pixels have been identified. On the other hand, if a pixel was noiseless, it will be categorized as without noise and other Filtering operations will be ignored so that the changes in details and tissues of the main image could be prevented.

\subsection{Recognition stage}

The image histogram is used by NAFSM Filter to estimate the intensity of the salt and pepper noise. The image contaminated with salt and pepper noise in the histogram creates two peaks and the operation begins based on the notion that these two peaks are related to noise. However, in many cases especially when the salt and pepper noise is not so much severe, the notion may not hold because the peaks may be related to those pixels without noise.In such cases, if the noise luminance is determined by histogram it would not hold and it makes the image noisy after filtering.

In order to resolve this problem, when the image's histogram is traveled toward a certain direction, the place of the first peak which we face is used to identify two local maximums $L$ salt and $L$ pepper which are two severities of salt-pepper noise. Searching two ends of the histogram is done by NAFSM. This search is sensitive to the edge and is directed toward the center of the histogram. The search is stopped when both local maximums $L_{\text {salt }}$ and $L_{\text {pepper }}$ are found. In general, for those images saved by 8 bit numbers, $L_{\text {salt }}=255$ and $L_{\text {pepper }}=0$ (figure2), these two severities of noise are used for recognition of noisy pixels in image. In order to determine the places of noisy pixels, the binary demonstration of noise, $N(i, j)$ is used:

$$
N(i, j)=\left\{\begin{array}{l}
1 \\
0
\end{array} \quad \begin{array}{l}
X(i, j)=L_{\text {sait }} \text { or } L_{\text {pepper }} \\
O . W
\end{array}\right.
$$

Where $x(i, j)$ is the value of the pixel in the place $(i, j)$ in the image. Thus, $N(i, j)=1$ indicated the pixel without noise which must be maintained and $N(i, j)=0$ indicate a noisy pixel which must be filtered.Figure (2) shows the histogram of the respective image (figure 6). 


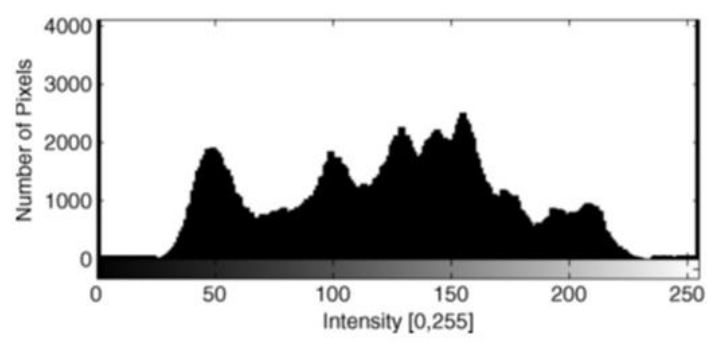

Figure 2: Lena image's histogram with $10 \%$ intensity of salt and pepper noise

\subsection{Filtering stage}

After the binary $\mathrm{N}$ is obtained, the noisy pixels $\mathrm{N}(\mathrm{i}, \mathrm{j})=0$ are replaced with their modified estimations. The NAFSM filter applies the square window $\mathrm{W}_{\mathrm{rs}}+(\mathrm{i}, \mathrm{j})$ with dimensions $(2 \mathrm{~s}+1) *(2 \mathrm{~s}+1)$.

$$
W_{2 s+1}(i, j)=\{X(i+m, j+n)\}
$$

where

$$
\mathrm{m}, \mathrm{n} \in(-\mathrm{s}, \ldots, \cdot, \ldots, \mathrm{s})
$$

Then, the number of pixel without noise $G_{2 s}+1(i, j)$ are identified as $W_{2 s}+1(i, j)$ as follows:

$$
G_{2 s+1}(i, j)=\sum_{m, n \in(-s, \ldots, 0, \ldots s)} N(i+m, j+n)
$$

If the window of the minimum filter $W_{2 s}+1(i, j)$ doesn't have pixels without noise (i.e. $\left.G_{2 s}+1(i, j)<1\right)$, the window of the filter is expanded and each four sides are added with one pixel $(s \rightarrow s+1)$ and this continues until the condition $G_{2 s}+1(i, j) \geq 1$ satisfies. For each identified noisy pixel, the size of filter's window is firstly set on $3 * 3$, i.e. $S=1$. The pixels without noise are used for selecting median pixel $M$ $(\mathrm{i}, \mathrm{j})$ :

$$
M(i, j)=\operatorname{median}\{X(i+m, j+n)\} \text { or } N(i+m, j+n)=1
$$

In order to select $\mathrm{M}$, only the pixels without noise are used so that selection of noisy pixels prevented for median pixels. However, the number of pixels without noise matters when selecting median pixel because higher number of pixels without noise take more times and an incorrect value for median pixel will be reached.

This subject has been shown together with using simulation on Lena image mixed with salt-pepper noise in figure 3 . These three dimensional diagrams show that the minimum number of pixels without pixels must be used so that the best value of the peak signal to noise ratio (PSNR) and the quickest time of calculations could be obtained. Similar diagrams could be obtained by using other sample image (figure 3). 


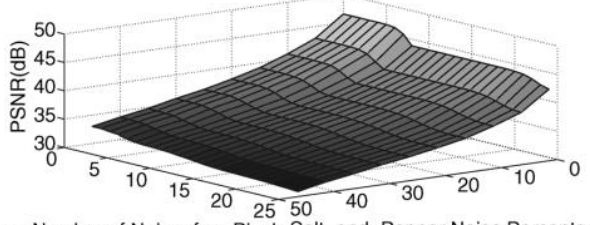

Minimum Number of Noise-free Pixels Salt-and-Pepper Noise Percentage (\%)

(a)

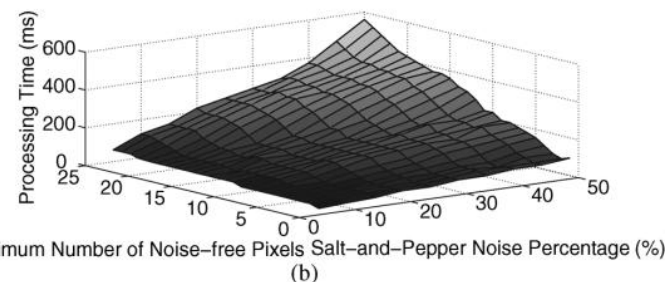

Figure(3): minimum number of pixels without noise required for selecting median pixel based on (a) PNSR and (b), processing time simulated for Lena image.

Since identification of noisy pixels is done based on measuring the intensity of luminance $L_{\text {salt }}$ and $L_{\text {pepper, }}$ The pixels without noise might be mistaken as noisy pixels in the areas with uniform intensity. Thus, the window of the filter must be permanently open and the selected median pixel may not be appropriate for it. For instance, consider the probability that When the window of the filter reaches to the size $7^{*} 7$ which means $s=3$, the search for pixels without noise may be stopped. It means the pixels without noise haven't been identified (i.e, $\left.G_{7}(l, j)=0\right)$. In this case, the first four pixels in the window $3 * 3$ is defined as follows:

$$
W_{3}(i, j)=\{X(i+k, j+l)\} \quad k, l \in(-1,0,1)
$$

To calculate it, the median pixel $M(i, j)$ will be used.

$$
M(i, j)=\text { median }\{X(i-1, j-1), X(i, j-1), X(i+1, j-1), X(i-1, j)\}
$$

Where $S=3$ and $G_{7}(i, j)=0$.

The first four pixels comprising the upper left part of the diameter of filter's window $3 * 3$ can be justified with respect to recursive feature of NAFSM filter. With respect to this process, each noisy pixel will be restored and recovered by pixels without noise due to the recursive behavior at the left upper part of the diameter of the filter's window. In consequence, application of four maximum pixels without noise instead of considering the entire neighbor pixels connected to it can make the median pixel more correct. After the median pixel, $m(i, j)$ has been found, the local information of the window $3 * 3$ could be found by absolute value ofdifferential luminance $d(i, j)=$

$$
d(i+k, j+l)=|X(i+k, j+l)-X(i, j)| \text { with }(\mathrm{i}+\mathrm{k}, \mathrm{j}+\mathrm{l}) \neq(\mathrm{i}, \mathrm{j})
$$

Then, the local information is defined as the maximum differential absolute value of luminance in the window $3 * 3=$

$$
D(i, j)=\max \{d(i+k, j+l)\}
$$


The process for selecting maximum operator in place of minimum operator is shown in figure a-4. The noise pixels are set with maximum luminance of 255 . This is while pixels without noise are considered within the variable interval. This indicates that such local information as the details of image, edges or pixels without noise have been considered for more processing. In contrast, the minimum operator cannot differentiate the pixels without noise from the noisy pixels. (e.g. image 4b).

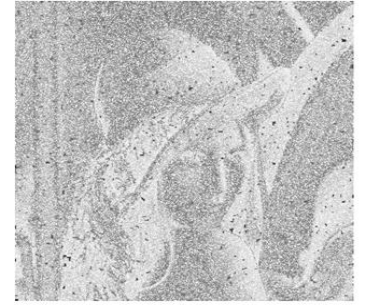

(a)

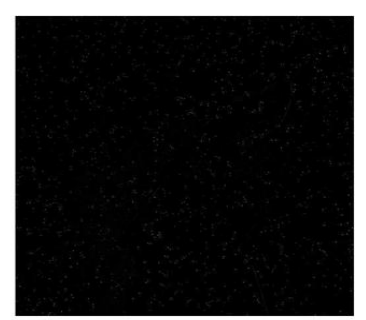

(b)

Figure 4: the local information derived from Lena image by using (a)- maximum operator and(b)- minimum operator.

As a part of filtering mechanism in NAFSM filter, the fuzzy inference is applied on local information D $(i, j)$ : the fuzzy series used in this research is shown in figure 5 and is defined as a function of fuzzy membership $F(i, j)$ as following:

$$
F(i, j)=\left\{\begin{array}{cc}
0 & D(i, j)<T_{1} \\
\frac{D(i, j)-T}{T_{2}-T_{1}} & T_{1}<D(i, j)<T_{2} \\
1 & D(i, j) \geq T_{2}
\end{array}\right.
$$

Where the local information $D(i, j)$ has been used as input fuzzy variable. In NAFSM filter, the threshold values of $T_{1}$ and $T_{2}$ are considered as constant and equal to 10 and 30 . In this thesis, these values are determined by PSO Algorithm.[8]

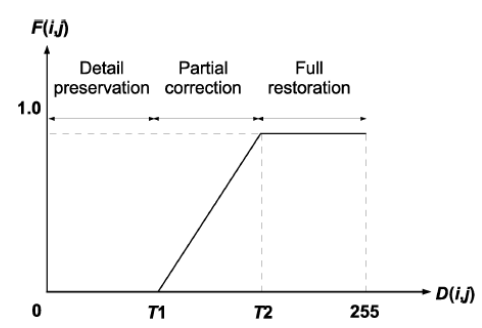

Figure 5- the fuzzy series used in NAFSM filter.

Finally, the corrective sentence is obtained as linear combination between the pixel under processing $X(i, j)$ and median pixel $M(i, j)$. The corrective sentence $Y(i, j)$ is given as follows.

$$
Y(i, j)=[1-F(i . j)], X(i, j)+F(i, j) \cdot M(i, j)
$$

Where the value of fuzzy membership $F(i, j)$ determines the weight for using $X(i, j)$ and $M(i, j)$. 


\section{Result of simulation}

\subsection{Result base on NAFSM filter}

In order to evaluate the deletion results, we define a criterion. The criterion is the ratio of total difference of the noisy deletion image $Y$ of the initial image I to the intensity of the initial image without noise. The less the value, the better the result will be.

$$
f=\frac{\sum|I-Y|}{\sum|I|}
$$

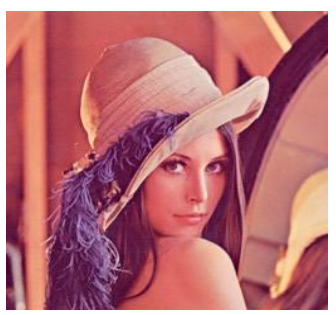

Figure (6): initial image without noise.

The initial image (figure 6 ) is formatted in grey and then noises with densities of $0.05,0.1,0.2$ and 0.5 are added. The standard values of $F$ for these simulations of NAFSM filter are $0.0027,0.0055$, 0.0111 and 0.0307 , respectively. Their results are shown in figure $7,8,9,10$, respectively.
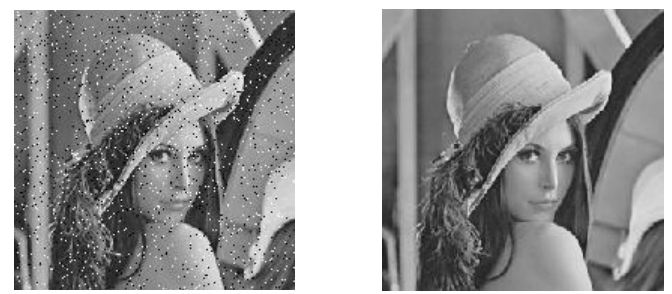

Figure 7: A noisy image with an intensity of 0.05 and the result of applying NAFSM filter.
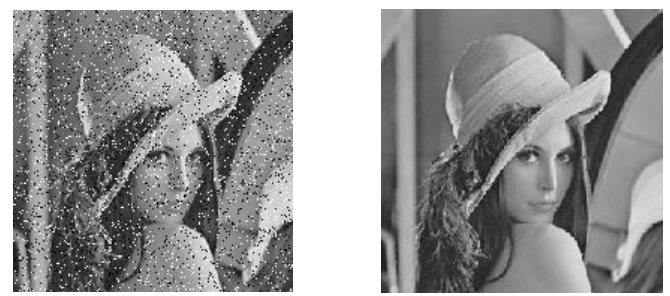

Figure (8): A noisy image with intensity of 0.1 and the result of applying NAFSM filter.
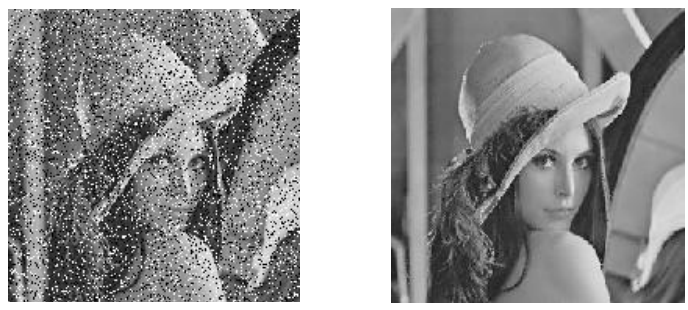
Figure (9): noisy image with intensity of 0.2 and the result of applying NAFSM filter.
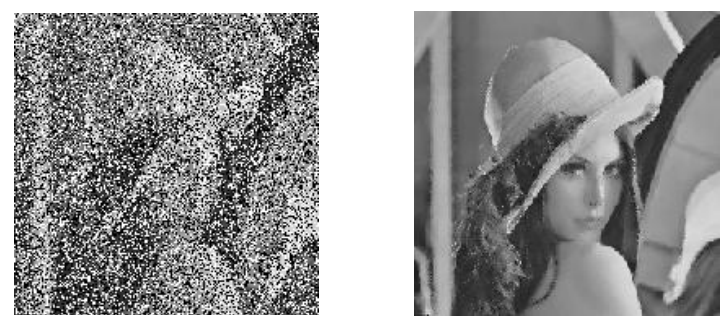

Figure (10): noisy image with intensity of 0.5 and result of applying NAFSM filter.

\subsection{Result based on fuzzy filter optimized by PSO}

Here, we will optimize the previous part by PSO algorithm: the parameters of the function for fuzzy membership $T_{1}$ and $T_{2}$ are optimized by PSO algorithm. The objective function is $F$ which should be minimized. For each solution, the objective function is calculated by applying the fuzzy filter on the noisy image. The best solutions are determined in each repetition and the places of the articles will be updated. Here, optimization is done by using 10 particles and 50 repetitions. Figure 11 outlines the curve for lowest value of $F$ in each repetition.

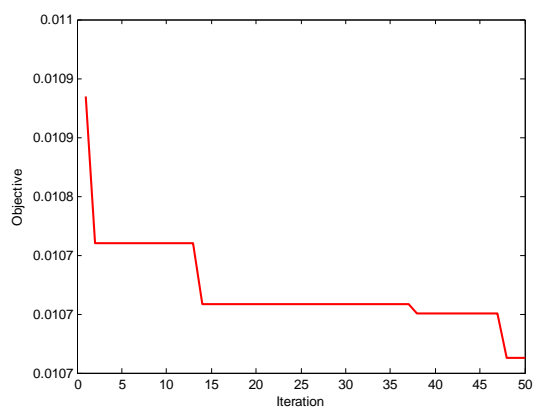

Figure 11: curve for the lowest value of $F$ in each repetition of PSO.

Figure 12 shows the deletion of noise with intensity of 0.2 by using the filter optimized by PSO. The value of $F$ for this result is equal to 0.01066 which shows a \%4 improvement $(0.0111)$ as nonoptimal mode.
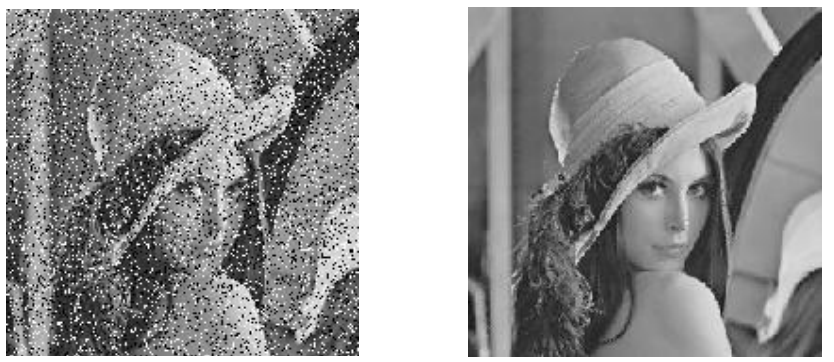

Figure12: noisy image with intensity of 0.2 and the result of applying optimized fuzzy filter. 
The results of optimized filter for deletion of intensities \%5, 0.10 and 0.5 are shown in figure 13, 14,15 . The values of $f$ equal $0.00263,0.00540$ and 0.0301 which show improvement of $\% 2.5$, $\% 1.8$ and $\% 1.19$, respectively.
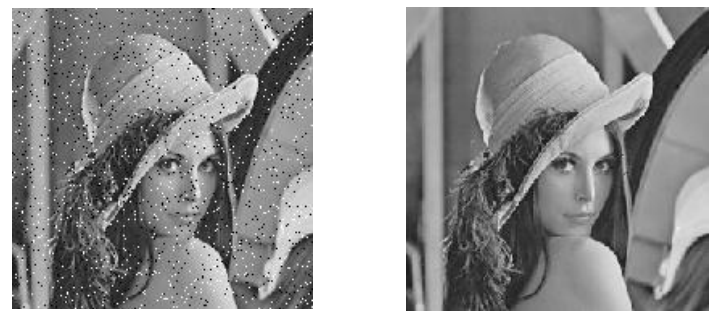

Figure (13): noisy image with intensity of $\% 5$ and the result of applying optimized fuzzy filter.
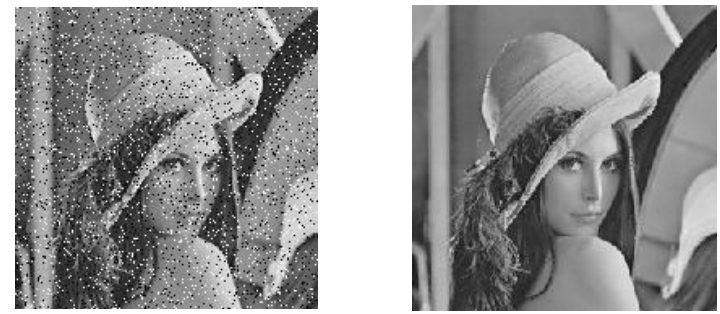

Figure (14): noisy image with intensity of 0.1 and the result of applying optimized fuzzy filter.
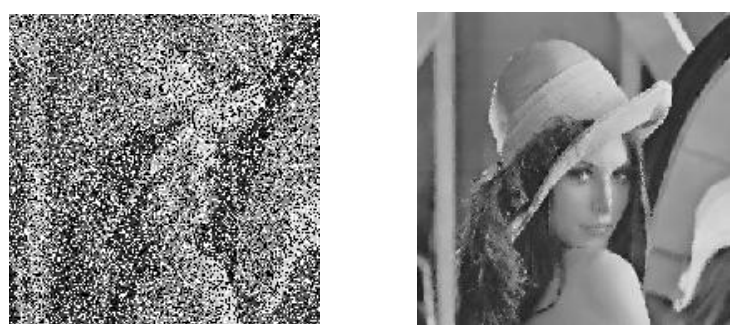

Figure(15): noisy image with intensity of 0.5 and the result of applying optimized fuzzy filter.

\subsection{Comparison of result}

In table 1 , the values of $f$ obtained for all filters and densities of the noises have been totally provided. Clearly, the filters have shown improved performances as compared to previous filter. Especially, the fuzzy filter optimized by PSO which is the recommended method of this article showed best results as compared to other filters. 
Tables 1- fitness values for performance of the filters in noise deletions with different intensities (densities).

\begin{tabular}{|c|c|c|c|c|}
\hline Filter & 0.05 & 0.1 & 0.2 & 0.5 \\
\hline Median filter & 0.0238 & 0.0269 & 0.0351 & 0.1494 \\
\hline Adaptive Median filter & 0.0071 & 0.0086 & 0.0143 & 0.1295 \\
\hline $\begin{array}{c}\text { Noise Adaptive Fuzzy } \\
\text { SwichingMedian } \\
\text { filter(NAFSM) }\end{array}$ & 0.0027 & 0.0055 & 0.0111 & 0.0307 \\
\hline $\begin{array}{c}\text { Noise Adaptive Fuzzy } \\
\text { SwichingMedian } \\
\text { filter(NAFSM)optimizedwith } \\
\text { PSO algorithm }\end{array}$ & 0.00263 & 0.0054 & 0.01066 & 0.0301 \\
\hline
\end{tabular}

Figure 16 shows the performance curve in terms of noise density for the simulated filters. It is seen that the conventional median filter is more sensitive than the noise density and its efficiency will be undesirable with increased noise intensity. However, other filters like filters optimized by PSO are less sensitive and show more uniform performances.

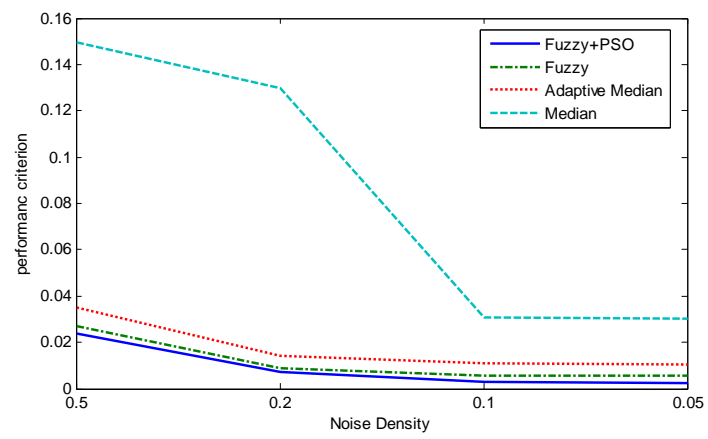

Figure (16): performance curve in terms of noise density for simulated filters.

\section{References}

[1] Zadeh, L.A., "Fuzzy sets," Information and Control, Vol. 8, pp. 338-353, 1965.

[2] J. Astola and P. Kuosmanen, Fundamentals of nonlinear digital filtering, Boca Raton, FL: CRC, 1997.

[3] Zadeh, L.A., "Outline of a new approach to the analysis of complex systems and decision processes,"

[4] Mamdani, E.H., "Applications of fuzzy logic to approximate reasoning using linguistic synthesis," IEEE

Transactions on Computers, Vol. 26, No. 12, pp. 1182-1191, 1977. 
[5] R. C. Eberhart and Y. Shi, "Particle swarm optimization: developments, applications and resources," in Proc. 2001 Congr. Evolutionary Computation, vol. 1, 2001.

[6] X. Chen and Y. Li, "A modified PSO structure resulting in high exploration ability with convergence guaranteed," IEEE Trans. Syst.,Man, Cybern. B, Cybern., vol. 37, no. 5, pp. 1271-1289, Oct. 2007.

[7] S. Kiranyaz, T. Ince, A. Yildirim and M. Gabbouj, "Fractional Particle Swarm Optimization in Multi Dimensional Search Space", IEEE Transactions on Systems, Man, and Cybernetics - Part B, in Press, doi:10.1016/j.neunet.2009.05.013, 2009.

[8] S. Gupta and S. Devi, Modified PSO Algorithm with High Exploration and Exploitation Ability, International Journal of Software Engineering Research \& Practices Vol.1, Issue 1, Jan, 2011.

[9] Rafael C. Gonzalez, Richard E. Woods, "Digital Image Processing", Pearson Prenctice Hall, 2007.

[10] Ronald R. Yager, Lotfi A. Zadeh, "An Introduction to Fuzzy Logic Applications in Intelligent Systems", Kluwer Academic Publishers Norwell, MA, 1992.

[11] Oscar Castillo and Patricia Melin, "Particle Swarm Optimization in the Design of Type-2 Fuzzy Systems", Recent Advances in Interval Type-2 Fuzzy Systems, Springer Briefs in Applied Sciences and Technology, 2012, Volume 1, 27-31.

[12] ZaferBingül, OğuzhanKarahan, “A Fuzzy Logic Controller tuned with PSO for 2 DOF robot trajectory control", Expert Systems with Applications Vol. 38, No. 1, Jan 2011, pp 1017-1031. 